\title{
Finerenone Dose-Exposure-Serum Potassium Response Analysis of FIDELIO-DKD Phase III: The Role of Dosing, Titration, and Inclusion Criteria
}

\author{
Sebastiaan Camiel Goulooze ${ }^{1} \cdot$ Nelleke Snelder $^{1} \cdot$ Andreas Seelmann $^{2} \cdot$ Andrea Horvat-Broecker $^{3} \cdot$ Meike Brinker $^{4}$. \\ Amer Joseph $^{4} \cdot$ Dirk Garmann $^{2} \cdot$ Joerg Lippert ${ }^{2} \cdot$ Thomas Eissing $^{2}$ (i)
}

Accepted: 8 October 2021 / Published online: 17 November 2021

(c) The Author(s) 2021

\begin{abstract}
Background Finerenone is a nonsteroidal selective mineralocorticoid receptor antagonist (MRA) that demonstrated efficacy in delaying the progression of chronic kidney disease (CKD) and reducing cardiovascular events in patients with CKD and type 2 diabetes mellitus in FIDELIO-DKD, where 5734 patients were randomized 1:1 to receive either finerenone or placebo, with a median follow-up of 2.6 years. Doses of finerenone 10 or $20 \mathrm{mg}$ once daily were titrated based on (serum) potassium and estimated glomerular filtration rate. The MRA mode of action increases potassium.

Methods Nonlinear mixed-effects population pharmacokinetic/pharmacodynamic models were used to analyze the finerenone dose-exposure-response relationship for potassium in FIDELIO-DKD. Individual time-varying exposures from pharmacokinetic analyses were related to the potassium response via a maximal effect, indirect-response model informed by 148,384 serum potassium measurements.

Results Although observed potassium levels decreased with increasing dose (i.e., inverse relation), model-based simulations for a fixed-dose setting (i.e., no dose titration) revealed the intrinsic finerenone dose-exposure-potassium response, with potassium levels increasing in a dose- and exposure-dependent manner, thus explaining the apparent conflict. The potassium limit for inclusion and uptitration from finerenone 10 to $20 \mathrm{mg}$ in FIDELIO-DKD was $\leq 4.8 \mathrm{mmol} / \mathrm{L}$. Modified limits of $\leq 5.0 \mathrm{mmol} / \mathrm{L}$ were simulated, resulting in higher hyperkalemia frequencies for both the finerenone and the placebo arms, whereas the relative hyperkalemia risk of a finerenone treatment compared with placebo did not increase.

Conclusions The analyses demonstrated the effectiveness of finerenone dose titration in managing serum potassium and provide a quantitative basis to guide safe clinical use.
\end{abstract}

\section{Introduction}

Finerenone is a nonsteroidal selective mineralocorticoid receptor antagonist (MRA) that recently demonstrated efficacy in delaying the progression of kidney disease and

Thomas Eissing

thomas.eissing@bayer.com

$1 \quad$ Leiden Experts on Advanced Pharmacokinetics and Pharmacodynamics (LAP\&P), Leiden, The Netherlands

2 Bayer AG, Pharmaceuticals R\&D, Pharmacometrics, Leverkusen/Wuppertal/Berlin, Germany

3 Bayer AG, Pharmaceuticals R\&D, Pharmacovigilance, Wuppertal, Germany

4 Bayer AG, Pharmaceuticals R\&D, Clinical Development, Wuppertal/Berlin, Germany reducing the risk of cardiovascular events in patients with chronic kidney disease (CKD) and type 2 diabetes (T2D) in the pivotal phase III trial FIDELIO-DKD (ClinicalTrials. gov number, NCT02540993) [1]. FIDELIO-DKD was an event-driven trial randomizing 5734 patients with advanced CKD to study kidney (primary) and cardiovascular (key secondary) outcomes. Design details of relevance for this manuscript are summarized in Sect. 2; further details have been published [2].

The efficacy and safety of finerenone is also investigated in three additional phase III studies: the recently completed FIGARO-DKD study (NCT02545049) [3] in patients with less advanced CKD and T2DM compared with patients in FIDELIO-DKD, FIND-CKD (NCT05047263) in patients with nondiabetic CKD, and FINEARTS-HF (NCT04435626) in patients with heart failure with a left ventricular ejection fraction of $\geq 40 \%$. 


\section{Key Points}

Mineralocorticoid receptor antagonists (MRAs) increase serum potassium. Finerenone-a nonsteroidal selective MRA - increased serum potassium and hyperkalemia events compared with placebo in the pivotal phase III study FIDELIO-DKD.

In this secondary model-based analyses, the dose-exposure-response relationship for serum potassium was quantified. The analyses demonstrated and explained a titration paradox, where higher finerenone doses were associated with lower serum potassium levels and lower incidences of hyperkalemia, based on serum potassiumguided dose adaption.

The impact of modified serum potassium inclusion and titration thresholds were simulated. The analyses demonstrated the effectiveness of finerenone dose personalization, supporting a favorable benefit-risk assessment and safe use in patients with chronic kidney disease and type 2 diabetes mellitus when guidance for dose and dose modification is followed.

Finerenone binds to the mineralocorticoid receptor (MR), which is expressed in many tissues and cells, including in the heart, kidneys, blood vessels, and immune cells, and blocks the effects of its natural hormone ligands, aldosterone and cortisol. Besides beneficial effects on inflammation and fibrosis, blockade of the MR at the collecting duct and distal nephron in the kidneys leads to a decreased reabsorption of sodium and consequently a decreased excretion of potassium into the urine. Thus, the use of finerenone cannot be devoid of any effect of increasing serum potassium $[4,5]$.

In the advanced CKD population studied in FIDELIODKD (mean estimated glomerular filtration rate [eGFR] $44.3 \mathrm{~mL} / \mathrm{min} / 1.73 \mathrm{~m}^{2}$, median urine albumin-to-creatinine ratio [UACR] $851 \mathrm{mg} / \mathrm{g}$ ) with a median follow-up of 2.6 years, hyperkalemia-related treatment-emergent adverse events (TEAEs; including Medical Dictionary for Regulatory Activities preferred terms "hyperkalemia" and "blood potassium increased") and serum potassium increases of $>5.5$ and $>6.0 \mathrm{mmol} / \mathrm{L}$ were collected. The number of subjects with hyperkalemia-related TEAEs was higher in the finerenone arm (18.3\%) than in the placebo arm (9.0\%) [1, $2]$. Most of these events were nonserious and mild or moderate in intensity. Hyperkalemia TEAEs leading to permanent discontinuation of study drug ( $2.3 \%$ of subjects) or hospitalization (1.4\% of subjects) constituted a small proportion of these events in the finerenone group. No fatal TEAEs of hyperkalemia were observed in either treatment group, and no evidence was found for an increased incidence of any severe clinical cardiac manifestations of hyperkalemia (e.g., ventricular arrhythmia or sudden cardiac death). The incidences of serum potassium $>5.5$ and $>6.0 \mathrm{mmol} / \mathrm{L}$ (based on central laboratory measurements) were 21.7 and $4.5 \%$ in the finerenone group and 9.8 and $1.4 \%$ in the placebo group, respectively [1].

Since assessment of hyperkalemia-related TEAEs was subject to the investigator's clinical judgment and provides limited binary information only, serum potassium was chosen to study the relation to finerenone exposure in a population pharmacokinetic and pharmacodynamic (popPKPD) model informed by more than 148,000 local and central serum potassium measurements. In this manuscript, serum potassium $>5.5$ and $>6.0 \mathrm{mmol} / \mathrm{L}$ are generally referred to as hyperkalemia of different severity.

We previously studied serum potassium popPKPD based on phase IIb data (ARTS-DN and ARTS-DN Japan studies) [6]. The analyses were extended to FIDELIO-DKD data to better understand the relationship between finerenone dose and exposure to serum potassium and hyperkalemia, qualitatively and quantitatively. Clinical trial simulations quantified the influence of serum potassium thresholds for inclusion and dose titration. The analyses also explained and highlighted the role of dose titration, which was implemented in phase III, in the management of serum potassium. In van den Berg et al. [7], we reported the population pharmacokinetic analysis of FIDELIO-DKD along with exposure-response (ER) analysis for the primary renal time-to-event endpoint. That analysis also provided the pharmacokinetic parameter post hoc estimates as a basis for the serum potassium popPKPD we describe here.

\section{Methods}

\subsection{Clinical Study}

Study design, patient characteristics, and the main study results have been published $[1,2,8]$. Information on informed consent, ethics, and analytical methods are also summarized in the electronic supplementary material (ESM).

In brief, FIDELIO-DKD was a randomized, doubleblind, placebo-controlled phase III study investigating the efficacy and safety of finerenone, in addition to standard of care $(\mathrm{SoC})$, on the progression of kidney disease in patients with T2DM and CKD. For inclusion, patients were required to have central laboratory serum potassium levels $\leq 4.8$ $\mathrm{mmol} / \mathrm{L}$ at both the run-in and the screening visits, although a single re-assessment was allowed for each visit. However, patients with serum potassium levels $>4.8 \mathrm{mmol} / \mathrm{L}$ at baseline ( $\geq 2$ weeks after screening) were allowed to start 
treatment at the investigator's discretion. The starting dose of finerenone was dependent on the eGFR values at screening visits. At randomization (baseline), subjects with an eGFR 25 to $<60 \mathrm{~mL} / \mathrm{min} / 1.73 \mathrm{~m}^{2}$ (central laboratory measurements) at the screening visits were assigned to the lower dose of finerenone $(10 \mathrm{mg})$ or placebo in addition to SoC, whereas subjects with an eGFR $\geq 60 \mathrm{~mL} / \mathrm{min} / 1.73 \mathrm{~m}^{2}$ were assigned to the higher dose of finerenone $(20 \mathrm{mg})$ or placebo in addition to SoC, which was the individually maximum tolerated labeled dose of either an angiotensin-converting enzyme inhibitor or an angiotensin II receptor blocker.

During the study, potassium was measured in the local laboratories of each study site-for titration and safety monitoring - as well as in the central laboratory. From the month 1 visit onwards, patients were eligible for uptitration of finerenone dose (from 10 to $20 \mathrm{mg}$ ) if local laboratory serum potassium levels were $\leq 4.8 \mathrm{mmol} / \mathrm{L}$ and if eGFR (local laboratory value) had not decreased by more than $30 \%$ compared with the value measured at the last regular visit. If local laboratory serum potassium levels were $>5.5$ $\mathrm{mmol} / \mathrm{L}$, study drug was interrupted and an additional re-test was scheduled within 3 days. After study drug interruption, subjects were allowed to re-start study drug at the lower dose of $10 \mathrm{mg}$ when serum potassium levels were $\leq 5.0 \mathrm{mmol} / \mathrm{L}$. Interruption of study drug was permitted at any time during the study for any safety reasons according to the investigator's clinical judgment. A safety visit for a local potassium assessment was scheduled \pm 4 weeks after each uptitration or re-initiation of treatment after an interruption of more than 7 days.

\subsection{Model Development}

A popPKPD model was developed to characterize the effect of finerenone exposure on serum potassium in NONMEM 7.4. The individual post hoc estimates of pharmacokinetic parameters from the FIDELIO-DKD population pharmacokinetic model were used to calculate the area under the finerenone concentration-time curve at steady state with the current dose level [7], which was used as the exposure metric to drive the effect of finerenone in the PKPD potassium model. Full simulated concentration-time profiles were also evaluated but did not improve the data description and were clearly computationally inferior. The pharmacokinetic model considered dose titration; with a half-life of $2.7 \mathrm{~h}$, a new pharmacokinetic steady state is rapidly attained $[6,7]$. As the model was supposed to simulate data for altered inclusion thresholds for serum potassium, data from screening failures (subjects who participated in run-in and screening visits but were not included in FIDELIO-DKD) were also considered for this analysis, with the exception of subjects who did not meet the eGFR inclusion criteria $(\geq 25$ and $<75$ $\mathrm{mL} / \mathrm{min} / 1.73 \mathrm{~m}^{2}$ at run-in and screening visits).
Model development occurred according to a stepwise approach, starting with the development of the structural model using the central laboratory data only. In the second and third steps, the local laboratory data were included and a covariate analysis performed, respectively. As a final step, potassium data from the run-in and screening visits (including data of screening failures) were added to the dataset. For nested models, during model development, additional parameters were only added to the model if this significantly $(p<0.001)$ improved the model fit, as determined by the objective function value ( -2 times the log-likelihood). If the models being compared were structurally different (and thus not nested), the models were compared using the Akaike information criterion.

The structural model included a turnover model for the effect of finerenone on serum potassium, similar to the phase IIb PKPD model [6]. The effect of finerenone was included as an effect on the first order dissipation rate constant $\left(k_{\text {out }}\right)$ parameter in the turnover model, and the following relationships were tested to characterize the ER relationship: linear, power, maximum drug effect $\left(E_{\max }\right)$, and sigmoid $E_{\max }$. The structural model development also tested the inclusion of a linear progression slope (TSLOPE) of serum potassium over time.

A limited number of known risk factors for hyperkalemia were tested as potential covariates on parameters for which interindividual variability was included in the model: age, baseline eGFR, baseline UACR, and sex. Based on diagnostic plots, the possible effect of baseline UACR and treatment randomization (active vs. placebo) were also tested on TSLOPE, even though interindividual variability for TSLOPE was not included in the model. Additionally, for comparability with a previously developed potassium PKPD model that included phase IIb data from a Japanese sister study (ARTS-DN Japan) [6], Japanese ethnicity was evaluated as a covariate for baseline serum potassium and residual variability.

\subsection{Model Evaluation}

The performance of the model was evaluated by performing simulations and comparing these with observations, hereafter referred to as a visual predictive check (VPC). The VPC simulations included residual error and interindividual variability.

Simulated subjects were "screened" at the run-in and screening visits according to the FIDELIO-DKD protocol criteria and removed from the simulated study if they did not meet the serum potassium inclusion criterion of $\leq 4.8$ $\mathrm{mmol} / \mathrm{L}$. To adequately reflect the FIDELIO-DKD dosing algorithm, the simulations for the VPC included simulation of dose titration decisions based on simulated local laboratory serum potassium observations. The occurrence 
of potassium re-tests and uptitration safety visits was also dependent on the simulated serum potassium observations and uptitration decisions, in line with the FIDELIO-DKD study design. Simulated central laboratory values were used to assess safety in terms of reaching serum potassium thresholds. A more detailed description of the VPC simulation workflow and computational methods and a NONMEM control stream are provided in the ESM.

\subsection{Potassium Threshold Simulations}

Additional simulations were performed to virtually explore the safety impact of increasing the serum potassium threshold from $\leq 4.8$ to $\leq 5.0 \mathrm{mmol} / \mathrm{L}$ to determine eligibility for inclusion and uptitration of finerenone. The same simulation workflow was used as to generate the simulated data for the VPCs, except that parameter uncertainty was included to reflect the whole estimated uncertainty, resulting in a scenario similar to that in FIDELIO-DKD (i.e., number of subjects, frequency of visits, duration of the study, covariate distribution, screening, and uptitration procedure). In line with the FIDELIO-DKD protocol, simulated local laboratory values were used to guide dose titration, and central laboratory values were used to describe safety results.

\section{Results}

\subsection{Clinical Study}

The analysis considered a total of 148,384 serum potassium observations (62,401 from local laboratory and 85,983 from central laboratory) from 10,070 subjects (5674 from the FIDELIO-DKD study population and 4396 from screening failures). From the FIDELIO-DKD randomized study population, there were a total of 141,138 serum potassium observations $(62,401$ from local laboratory and 78,737 from central laboratory), of which 117,063 were post-baseline serum potassium observations. Of the 5674 subjects valid for analysis in the FIDELIO-DKD study population, 2841 were randomized to placebo and 2833 to active treatment (2622 of which started on the starting dose of $10 \mathrm{mg}$ and 211 on the dose of $20 \mathrm{mg}$ ). The median (5th-95th percentiles) eGFR and UACR at baseline in FIDELIO-DKD was 43.0 (26.7-66.9) $\mathrm{mL} / \mathrm{min} / 1.73 \mathrm{~m}^{2}$ and 852 (140-3366) $\mathrm{mg} / \mathrm{g}$, respectively. The average dose level over time was $15.1 \mathrm{mg}$ [1]. Of the post-baseline serum potassium observations, $34.8 \%$ were at the $10 \mathrm{mg}$ dose level, $48.7 \%$ at the $20 \mathrm{mg}$ dose level, and $16.5 \%$ during (permanent or temporary) treatment interruption (Fig. 1a).

Dose-response analysis for the longitudinal potassium data was hampered by the nonconstant dosing over time. To get a first overview, we grouped serum potassium measurements based on the dose level prior to measurement. This means that, for example, a subject's potassium value may have been assigned to the $10 \mathrm{mg}$ dose group at one instance but to the $20 \mathrm{mg}$ dose group subsequently, if the patient was uptitrated in between. Higher serum potassium levels and frequencies of serum potassium $>5.5$ or $>6.0$ $\mathrm{mmol} / \mathrm{L}$ were observed in the active treatment arm than in the placebo arm. However, there was an inverse correlation between the serum potassium levels and the finerenone dose level (Fig. 1a, Pearson correlation test within active dose group: $-0.161 ; 95 \%$ confidence interval -0.172 to -0.151 ; $\left.p<2.2 \mathrm{e}^{-16}\right)$. The highest incidences of serum potassium values $>5.5$ or $>6.0 \mathrm{mmol} / \mathrm{L}$ were in patients in the active treatment arm during treatment interruption or discontinuation, and patients receiving $10 \mathrm{mg}$ had an approximately twofold higher incidence of hyperkalemia than patients receiving $20 \mathrm{mg}$. A visual inspection showed that the inverse correlation was also observed for absolute serum potassium values versus finerenone exposure but not for potassium change from baseline (see Figs. S1 and S2 in the ESM). This indicated that serum potassium-guided dose titration was driving the inverse correlation seen for absolute values of potassium versus exposure and dose. To test the hypothesis and quantitatively characterize the exposure versus serum potassium response, a popPKPD model was established.

\subsection{Model Development}

After stepwise model development, a model characterizing the finerenone ER relationship for serum potassium was successfully developed. Final model parameter estimates are shown in Table 1, and covariate equations and final model code are provided in the ESM. An $E_{\max }$ function best described the effect of finerenone on serum potassium on $k_{\text {out }}$, the parameter in the turnover model that was used to characterize the time-to-steady state potassium after disruption of potassium homeostasis by the finerenone effect. The typical value of the $E_{\text {max }}$ on $k_{\text {out }}$ in the turnover model corresponded to an increase in serum potassium of $9.95 \%$, which amounted to an increase of $0.44 \mathrm{mmol} / \mathrm{L}$ for a patient with a serum potassium baseline of $4.4 \mathrm{mmol} / \mathrm{L}$. The typical effect at 10 and $20 \mathrm{mg}$ in the model was an increase in serum potassium of 3.86 and $5.56 \%$, respectively. For a subject with a serum potassium baseline of $4.4 \mathrm{mmol}$, this corresponded to an increase of 0.17 and $0.25 \mathrm{mmol} / \mathrm{L}$ for 10 and $20 \mathrm{mg}$, respectively; however, the increase appeared dose independent in the observed data (see Fig. 1b). The model also included a disease progression slope that increased the predicted serum potassium levels over time, with a $16 \%$ faster rate of progression in patients with each $0.1 \mathrm{mmol} / \mathrm{L}$ increase in predicted baseline serum potassium and a $61 \%$ lower rate of progression in subjects in the active treatment arm compared with the placebo arm. 
A

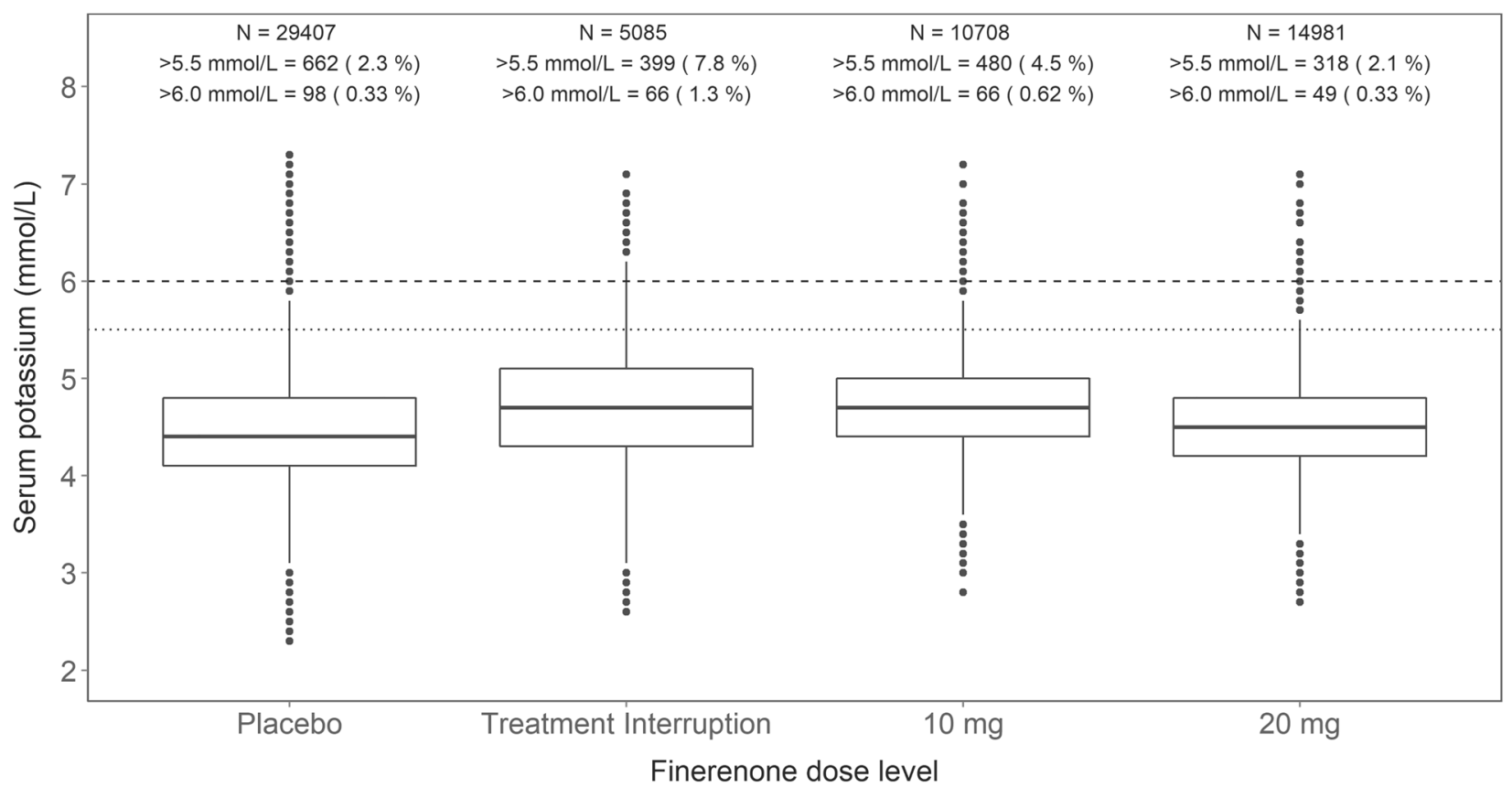

B

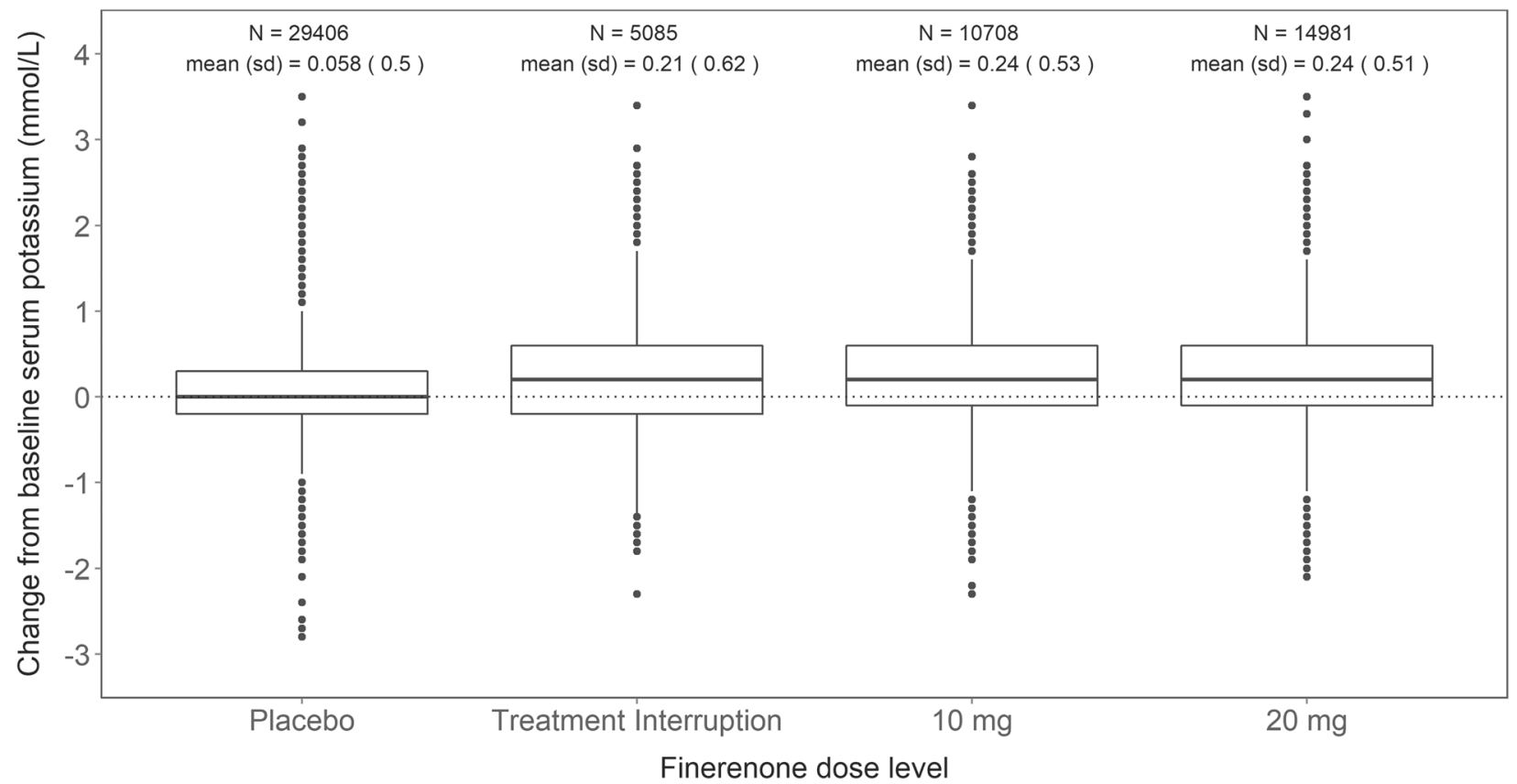

Fig. 1 Finerenone dose-response on serum potassium. Boxplots of central laboratory $\mathbf{a}$ absolute and $\mathbf{b}$ change from baseline serum potassium levels after the baseline visit. The column 'placebo' arm shows all data for subjects in the placebo arm. Stratification of the other three columns for the active treatment arm are based on the actual dose level of the treatment at the time of the serum potassium measurement. The column 'treatment interruption' shows data for subjects in the active treatment arm who had treatment interrupted or discontinued (permanently). Dotted and dashed horizontal reference lines at 5.5 and $6.0 \mathrm{mmol} / \mathrm{L}$, respectively. $s d$ standard deviation 
During the covariate analysis, the following effects were identified: baseline eGFR (3.98\% higher serum potassium baseline and $32 \%$ higher $E_{\max }$ at 5 th percentile vs. 95 th percentile of eGFR distribution), baseline UACR (24\% lower $E_{\max }$ and $94 \%$ lower rate of progression at 5th percentile vs. 95th percentile of UACR distribution), sex (14.3\% lower $E_{\max }$ in females), and Japanese ethnicity (3.62\% lower baseline serum potassium and $13 \%$ lower standard deviation of residual error).

The final potassium model successfully captured both serum potassium levels over time in the study duration (Fig. 2) and the percentage of patients with one or more serum potassium observations $>5.5$ or $6.0 \mathrm{mmol} / \mathrm{L}$ (Fig. 3). Additionally, the doses predicted by the dose titration simulations matched the observed dosing patterns in FIDELIODKD (Fig. 4).

A comparison between simulations of the model accounting for dose titration and simulations assuming constant dose levels provided strong support for the above hypothesis. The simulations considering dose titration closely resembled the apparent inverse dose response (Fig. 5b), whereas the simulations assuming a constant dose over time, i.e., without any dose titration, illustrated the underlying ER, where serum potassium increased in an exposure- and dose-dependent manner (Fig. 5a).

\subsection{Potassium Threshold Simulations}

The simulated impact of virtually increasing the serum potassium thresholds for inclusion and uptitration in a FIDELIO-DKD-like scenario (clinical trial simulation) is reported in Table 2. The increase of the inclusion threshold from $\leq 4.8$ to $\leq 5.0 \mathrm{mmol} / \mathrm{L}$ increased the fraction of screened patients eligible for inclusion based on potassium from 81.8 to $91.3 \%$. The less restrictive thresholds were predicted to result in an increase in the percentage of patients with one or more hyperkalemia occurrences (potassium $>5.5 \mathrm{mmol} / \mathrm{L}$ ) from 20.3 to $24.0 \%$ in the finerenone group. This can be primarily attributed to the change of the inclusion threshold, as the impact of changing the uptitration
Table 1 Parameter estimates and uncertainties of the final PKPD serum potassium model

\begin{tabular}{|c|c|c|}
\hline Parameter name & Estimate & $\operatorname{RSE}(\%)$ \\
\hline$\theta_{\text {pop, BSL }}$, BSL (mmol/L) & 4.50 & 0.140 \\
\hline$\theta_{\text {pop,local }}$, relative difference local lab vs. central lab & 0.0204 & 2.25 \\
\hline$\theta_{\text {pop,kin }}, k_{\text {in }}(\mathrm{mmol} / \mathrm{L} / \mathrm{h})$ & 0.00981 & 14.2 \\
\hline$\theta_{\text {pop,EMAX }}, E_{\max }$ & 0.0905 & 16.2 \\
\hline$\theta_{\text {pop,EC50 }}, \mathrm{EC} 50(\mathrm{mg} \times \mathrm{h} / \mathrm{L})$ & 0.512 & 33.3 \\
\hline$\theta_{\text {pop,TSLOPE,placebo }}$, TSLOPE in placebo arm (/year) & 0.00412 & 14.2 \\
\hline$\theta_{\text {pop, TSLOPE,active }}$, TSLOPE in active treatment arm (/year) & 0.00161 & 24.9 \\
\hline$\theta_{\mathrm{JAP}, \sigma}$, relative $\sigma$ with Japanese ethnicity (\%) & 87.0 & 3.64 \\
\hline$\theta_{\mathrm{JAP}, \mathrm{BSL}}$, relative difference in BSL with Japanese ethnicity (\%) & -3.62 & 10.1 \\
\hline$\theta_{\text {EGFR,BSL}}$, effect of EGFREPI0 on BSL & -0.0429 & 8.02 \\
\hline$\theta_{\mathrm{BSL}, \mathrm{TSLOPE}}$, effect of BSL on TSLOPE (L/mmol) & 1.60 & 10.8 \\
\hline$\theta_{\mathrm{UACR}, \mathrm{TSLOPE}}$, effect of UACR0 on TSLOPE $(\mathrm{g} / \mathrm{mg})$ & 0.00114 & 19.6 \\
\hline$\theta_{\text {EGFR,EMAX }}$, effect of EGFREPI0 on $E_{\max }$ & -0.305 & 23.6 \\
\hline$\theta_{\text {UACR,EMAX }}$, effect of UACR0 on $E_{\max }(\mathrm{g} / \mathrm{mg})$ & 0.0000931 & 23.7 \\
\hline$\theta_{\text {SEX,EMAX }}$, effect of sex on EMAX & -0.143 & 20.1 \\
\hline \multicolumn{3}{|l|}{ Interindividual variability } \\
\hline $\begin{array}{l}\theta_{\text {boxcox,IIV,BSL }} \text {, shape parameter box-cox transformation exponential IIV } \\
\text { on BSL }\end{array}$ & -1.61 & 17.4 \\
\hline$\omega^{2}$ exponential BSL & 0.00717 & 2.43 \\
\hline$\omega^{2}$ proportional $E_{\max }$ & 1.49 & 10.2 \\
\hline$\omega^{2}$ covariance BSL/ $E_{\max }$ & -0.0385 & 10.8 \\
\hline \multicolumn{3}{|l|}{ Residual error } \\
\hline$\sigma^{2}$, scalar of residual error & 0.00447 & 0.986 \\
\hline$v$, degrees of freedom of $t$-distributed residual error & 6.60 & 1.81 \\
\hline
\end{tabular}

$B S L$ baseline serum potassium, $E C 50$ exposure (in $\mathrm{AUC}_{\tau, \mathrm{MD}}$ ) at which the effect is $50 \%$ of the maximum effect, EGFREPIO estimated glomerular filtration rate at baseline, $E_{\max }$ maximum effect, $k_{\text {in }}$ zero-order production rate of serum potassium in turnover model, $P K P D$ pharmacokinetic pharmacodynamic, $R S E$ relative standard error of estimate, TSLOPE progression rate, UACRO urine albumin-to-creatinine ratio at baseline 


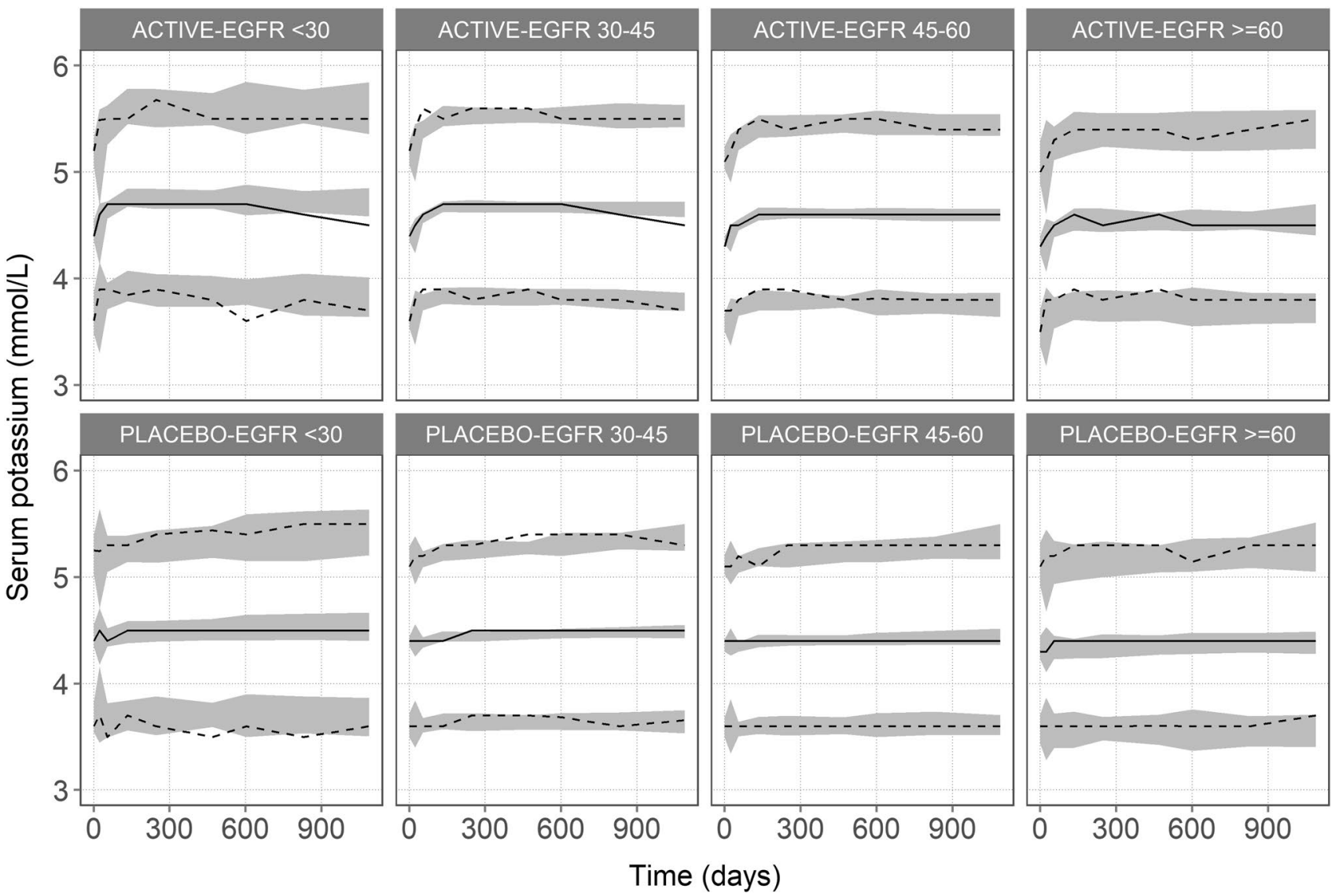

Fig. 2 Visual predictive check of central laboratory serum potassium data over time, stratified by estimated glomerular filtration rate $\left(\mathrm{eGFR} ; \mathrm{mL} / \mathrm{min} / 1.73 \mathrm{~m}^{2}\right.$ ) category. The black lines show the observed median (solid line) and 5th and 95th percentile (dashed

threshold was predicted to be limited (data not shown). In addition, the threshold increase was predicted to lead to an increase in the frequency of dose interruptions from 8.5 to $11.2 \%$ in the finerenone group. Although the absolute risk increased, the relative risk of hyperkalemia with finerenone treatment compared with placebo reduced because of a disproportional increase in the absolute hyperkalemia risk in the placebo group.

Looking separately at the subset of patients who were included when the inclusion threshold was increased from $\leq 4.8$ to $\leq 5.0 \mathrm{mmol} / \mathrm{L}$ (9.5\% of screened patients), a high hyperkalemia risk for both the active treatment (51\% of subjects with serum potassium $>5.5 \mathrm{mmol} / \mathrm{L}$ ) and placebo arms $(42.9 \%$ of subjects with serum potassium $>5.5 \mathrm{mmol} / \mathrm{L})$ was simulated. However, this subset had a lower relative risk of serum potassium $>5.5 \mathrm{mmol} / \mathrm{L}$ with finerenone treatment (1.2-fold higher than those receiving placebo) compared with the whole simulated population (with serum potassium $\leq 5.0 \mathrm{mmol} / \mathrm{L}$ ) in this scenario (1.87-fold higher than those receiving placebo). lines), and the gray areas indicate the $99 \%$ prediction interval of the same percentiles in the simulations, which include variability but not parameter uncertainty

\section{Discussion}

Finerenone, with its inherent mode of action as an MRA, increases serum potassium, particularly in patients with advanced CKD who are on maximum tolerated labeled doses of a renin-angiotensin system inhibitor, as studied in FIDELIO-DKD. Accordingly, the analysis showed increased serum potassium levels and hyperkalemia rates with finerenone treatment compared with placebo. However, the analysis of serum potassium values and finerenone exposure or dose revealed an apparent inverse relationship not in line with a naïve "traditional" assumption, where the highest hyperkalemia risk would be expected at the highest dose level (Fig. 1).

The model-based analysis can explain this phenomenon. In this model, finerenone increased serum potassium levels in an exposure-dependent fashion, with the strongest effect being predicted at the higher dose level $(20 \mathrm{mg})$, as shown in Fig. 5a illustrating the intrinsic dose-response relationship, i.e., the relationship with 


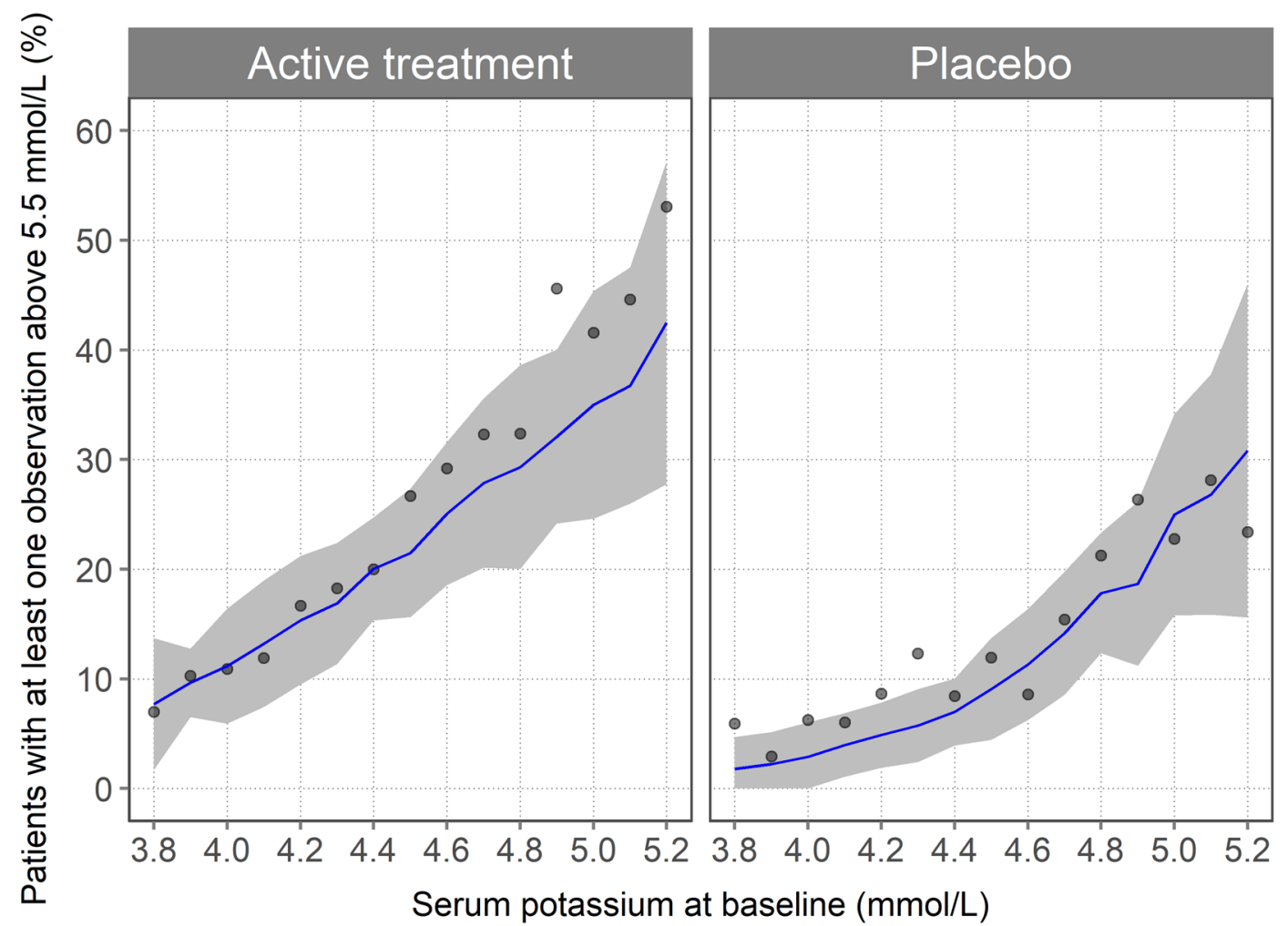

A Hyperkalemia $>5.5 \mathrm{mmol} / \mathrm{L}$ versus baseline serum potassium

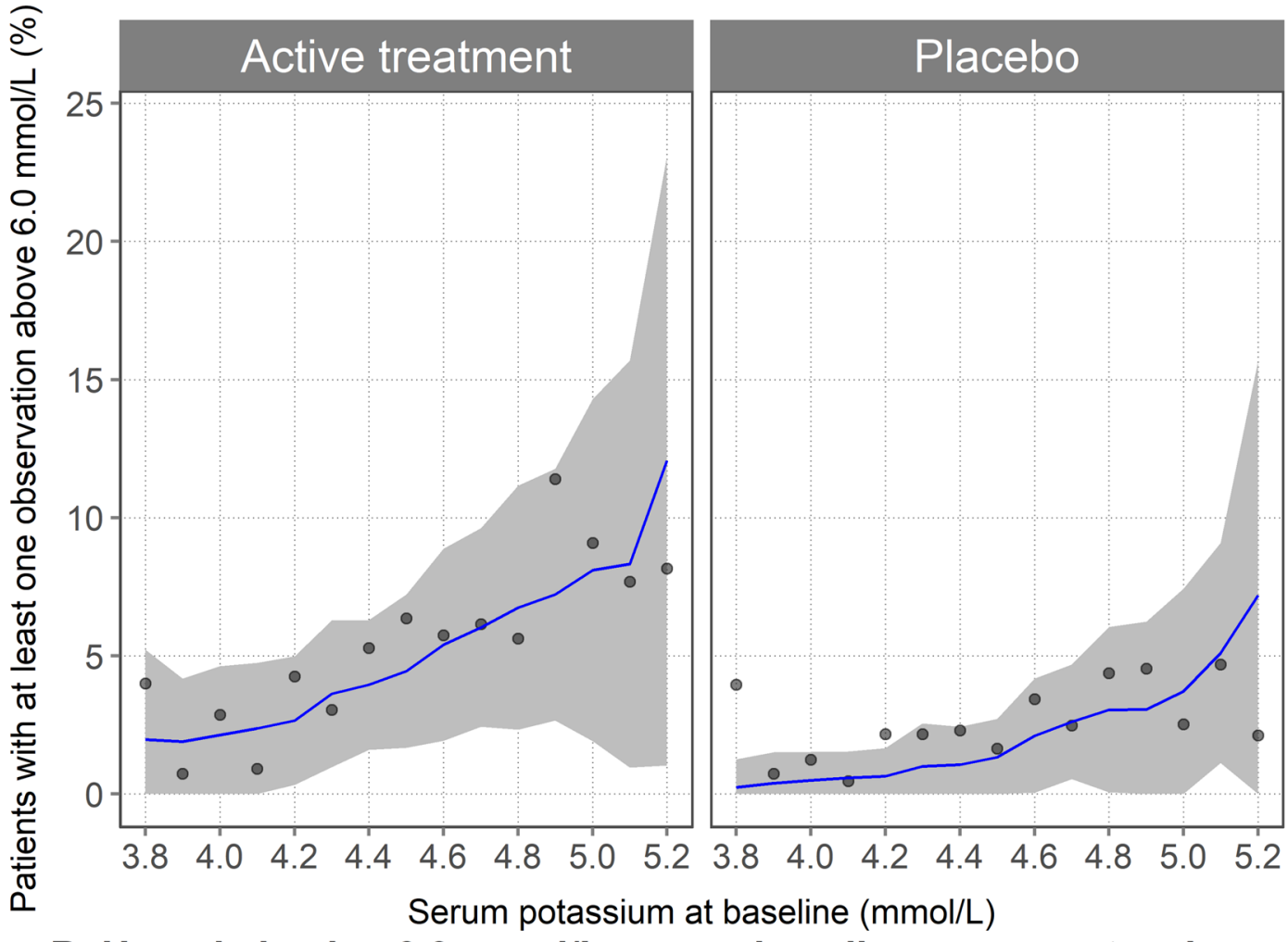

B Hyperkalemia $>6.0 \mathrm{mmol} / \mathrm{L}$ versus baseline serum potassium 
4 Fig. 3 Visual predictive check of percentage of patients with one or more serum potassium observations $>5.5$ and $6.0 \mathrm{mmol} / \mathrm{L}$ vs. baseline serum potassium. Simulated data include residual error and interindividual variability (but not parameter uncertainty) and are shown as mean (gray lines) and 95\% prediction interval (gray area). Observed data are shown as black points. Only data from patients with serum potassium baseline between 3.8 and $5.2 \mathrm{mmol} / \mathrm{L}$ are included in this figure, as the number of patients outside this range was too low to allow calculation of the percentage of hyperkalemia with reasonable precision

a constant dose assuming no dose titration. However, patients whose serum potassium levels were too high discontinued or interrupted treatment and re-started at the lower dose level, whereas patients with lower serum potassium levels were uptitrated to the higher dose. By simulating the dose-titration decisions based on simulated serum potassium levels, the model re-created the pattern in the observations where the patients on the higher dose level had the lower serum potassium levels as shown in Fig. 5b. Although dose titration was a design element of FIDELIODKD intended to manage hyperkalemia, it inverted the relation between dose and serum potassium, which may not have been expected a priori. Although the risk for an individual patient followed the intrinsic dose-response relationship and thus increased with dose, in the apparent behavior of the FIDELIO-DKD population, patients receiving higher doses had a lower hyperkalemia risk compared with patients receiving a lower dose. Interestingly, independent of the findings described here, Schnider et al. [9] also recently described and conceptually proved in a related setting how dose titration can lead to the described phenomenon, referring to the "drug titration paradox," and related work providing hints has been published [10-12]. From a systems theory perspective, dose titration introduces negative feedback, which is the basis of control and supports stabilization of systems, i.e., reducing fluctuations and supporting equilibration. The concept is wellestablished for various biological systems and, in physiology, is often relevant to the support of homeostasis, including in the face of perturbations [13-15].

Besides providing qualitative insight into serum potassium dynamics under finerenone treatment, the model-based covariate analysis quantified the effect of important risk factors. Most prominently, in the model, patients with low eGFR and/or high UACR had an increased risk of hyperkalemia because of effects on baseline serum potassium, finerenone effect, or disease progression. The identified effects were in line with general expectations, as low eGFR and high UACR characterize disease progression and are known to be associated with an increased risk for hyperkalemia [16-18]. The number of covariates tested in the current study was limited, and evaluating additional factors such as comedications or comorbidities in the future could be of interest to further support the understanding of serum potassium biology and hyperkalemia risk.

In FIDELIO-DKD, patients who received finerenone had higher mean serum potassium levels than those who received placebo. A maximum mean difference between both groups, of $0.23 \mathrm{mmol} / \mathrm{L}$, was observed at month 4 , and the difference remained largely stable thereafter [1]. With a slower disease progression rate in patients receiving finerenone than in those receiving placebo, the current analysis also identified an opposite, i.e., lowering, effect on potassium on the long term. This potassium-lowering effect likely reflected the kidney-protective effect of finerenone treatment $[1,7]$. Although the net effect on the mean potassium values was an increase with finerenone treatment within the timescale investigated in FIDELIO-DKD (median follow-up 2.6 years), the effect on potassium lowering may attenuate the potassium-increasing effect of finerenone compared with placebo during long-term treatment.

In FIDELIO-DKD, serum potassium $\leq 4.8 \mathrm{mmol} / \mathrm{L}$ was required for inclusion into the study, based on values at run-in and screening. Similarly, dose uptitration was limited to subjects with values below that threshold. However, because of the variability in serum potassium, $13.6 \%$ of subjects at baseline (randomization) had a serum potassium level $>4.8 \mathrm{mmol} / \mathrm{L}$. Variability in the model was considered random and reflected the unknown effects of, for example, amounts of potassium in food or patient hydration status. These subjects also informed the model on treatment effects with high baseline values. Generally, the model adequately described the potassium dynamics and treatment effect, including pretreatment, start, uptitration, interruption, or discontinuation (example shown in Fig. 2). Therefore, the model was considered suitable to assess the influences of increased inclusion or uptitration thresholds. Model-based analyses showed that the absolute risk for hyperkalemia increased with increasing baseline serum potassium values, whereas the risk ratio of hyperkalemia for patients receiving finerenone relative to those receiving placebo did not (but rather decreased according to the model), because the hyperkalemia risk increased disproportionately for patients receiving placebo compared with patients receiving finerenone (Fig. 3). This was also seen in simulations with a FIDELIO-DKD-like population, where inclusion and uptitration limits (serum potassium $\leq 4.8 \mathrm{mmol} / \mathrm{L}$ ) were compared with simulations of an extended scenario with these limits increased to serum potassium $\leq 5.0 \mathrm{mmol} / \mathrm{L}$ (Table 2 ). The absolute risk for hyperkalemia also (substantially) increased in the subset of this population with serum potassium between $>4.8$ and $\leq 5.0 \mathrm{mmol} / \mathrm{L}$, whereas the risk ratio decreased compared with the FIDELIO-DKD setting. Based on these findings, 


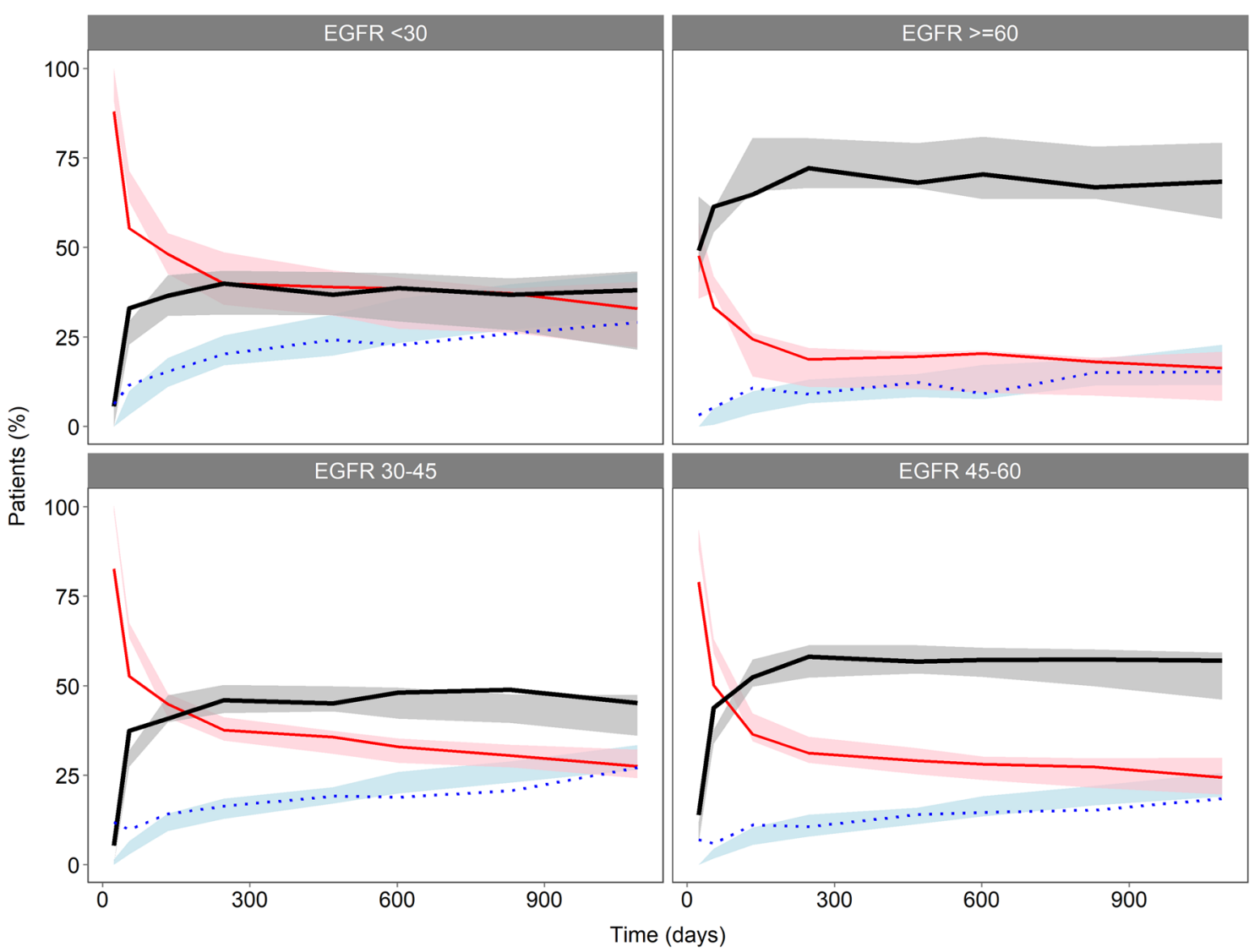

Fig. 4 Visual predictive check of finerenone dosing in dose-titration simulations for the final pharmacokinetic/pharmacodynamic model, stratified by baseline estimated glomerular filtration rate (eGFR; mL/ $\min / 1.73 \mathrm{~m}^{2}$ ) category. The lines indicate the observed frequency of the dose levels of $20 \mathrm{mg}$ (solid black) or $10 \mathrm{mg}$ (solid red) over time after the first baseline visit. The observed frequency of patients on

model-based analyses indicated that patients with high serum potassium baseline values, especially $>4.8 \mathrm{mmol}$, had a high risk for hyperkalemia independent of treatment assignment, and the risk increased disproportionately in the placebo group. FIDELIO-DKD studied a population with advanced CKD, but very recently published FIGARODKD data indicated a lower finerenone-related absolute hyperkalemia risk in patients with better preserved renal function [3].

FIDELIO-DKD was a population at increased risk of hyperkalemia, as also shown by the hyperkalemia rates in patients receiving placebo, where the use of steroidal MRAs is typically avoided [19], or in case of eplerenone contraindicated (US and UK prescribing information). A meta-analysis indicated a fourfold increase in hyperkalemia with steroidal MRAs in CKD with T2DM [20], whereas the data for finerenone presented in Sects. 1 and 3 point more towards a twofold increase. permanent or temporary treatment interruption is shown as a dotted blue line. The colored areas indicate the $99 \%$ prediction interval of the dose level percentages in the simulations, which include variability but not parameter uncertainty. Patients in the placebo arm are not included in this figure

\section{Conclusion}

Our analyses showed how negative feedback via serum potassium-guided dose titration inversed the intrinsic finerenone ER relationship for serum potassium and led to an apparent (counterintuitive) dose response, where subjects receiving the higher dose had the lower serum potassium values and lower hyperkalemia risk. Analyses of data collected in the context of dose titration and interruption indicated that hyperkalemia under finerenone treatment was manageable using a dose-titration regimen based on serum potassium and eGFR. The effectiveness of this regimen, also accounting for extrinsic and intrinsic factors, is reflected in the US prescribing information [21]. Simulations to quantify the risk of increased inclusion or titration limits for serum potassium were also relevant for dosing guidance in the label [21]. Together with the (primary) efficacy analyses $[1,7]$, the findings support a favorable 


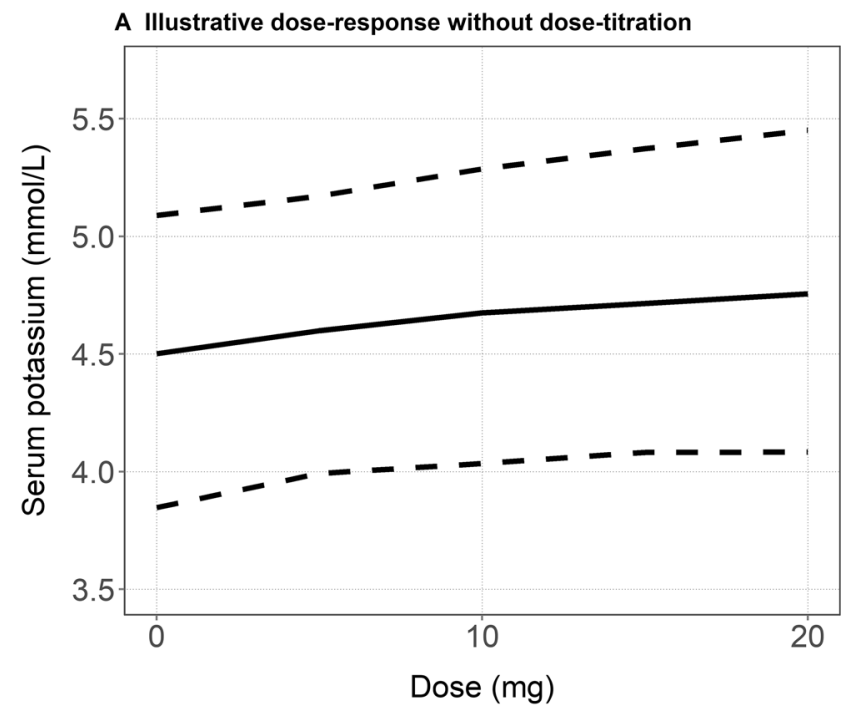

Fig. 5 a Intrinsic dose-response (i.e., without dose titration) of finerenone and the $\mathbf{b}$ impact of dose titration on the observed doseresponse of finerenone on serum potassium in FIDELIO-DKD. a The dose-response relationship without accounting for dose titration. Shown are the median and $90 \%$ prediction interval of 10,000 simulated individual predicted serum potassium levels (including interindividual variability, but not residual error) with drug effect at steady state, ignoring the impact of disease progression. All simulated patients have typical covariates (male sex, non-Japanese ethnicity,

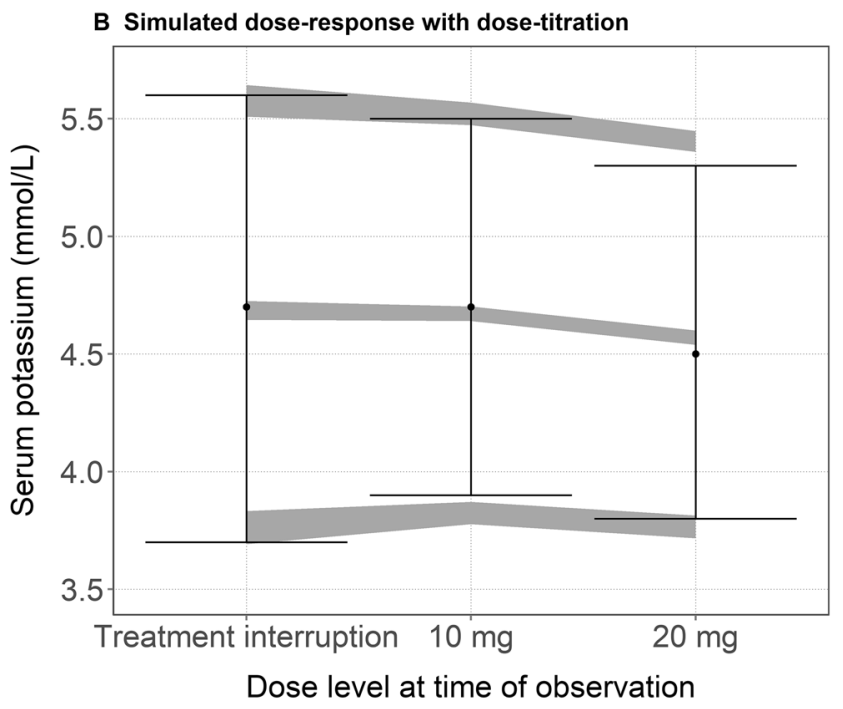

baseline estimated glomerular filtration rate of $45 \mathrm{~mL} / \mathrm{min} / 1.73 \mathrm{~m}^{2}$, baseline urine albumin-to-creatinine ratio of $800 \mathrm{mg} / \mathrm{g}$, and a typical finerenone clearance of $28.0 \mathrm{~L} / \mathrm{h}$ ). b Visual predictive check of the dose-response of finerenone on serum potassium. Observed data are shown as black points (median) and error bars (depicting the 5th and 95th percentiles), and the gray areas indicate the $99 \%$ variabilitybased prediction interval of simulations that include dose titration (excluding parameter uncertainty)

Table 2 Summary of influence of serum potassium thresholds for inclusion and uptitration limits in a FIDELIO-DKD-like simulation scenario

\begin{tabular}{llrr}
\hline Hyperkalemia & $\begin{array}{l}\text { Total population } \leq 4.8 \mathrm{mmol} / \mathrm{L} \\
\text { limits scenario }\end{array}$ & $\begin{array}{l}\text { Total population } \leq 5.0 \mathrm{mmol} / \mathrm{L} \\
\text { limits scenario }\end{array}$ & $\begin{array}{l}\text { Subset only included } \\
\text { in } \leq 5.0 \mathrm{mmol} / \mathrm{L} \text { limit } \\
\text { scenario }\end{array}$ \\
\hline$>5.5 \mathrm{mmol} / \mathrm{L}$ on finerenone $(\%)$ & $20.3(17.9-22.6)$ & $24.0(21.2-26.8)$ & $51.0(45.2-56.8)$ \\
$>5.5 \mathrm{mmol} / \mathrm{L}$ on placebo $(\%)$ & $9.42(7.99-10.9)$ & $12.9(11.4-14.3)$ & $42.9(37.7-48.0)$ \\
$>5.5 \mathrm{mmol} / \mathrm{L}$ RR $(-)$ & $2.16(1.76-2.56)$ & $1.87(1.58-2.16)$ & $1.20(0.982-1.41)$ \\
$>6.0 \mathrm{mmol} / \mathrm{L}$ on finerenone $(\%)$ & $4.50(3.61-5.39)$ & $5.88(4.91-6.85)$ & $15.0(10.5-19.4)$ \\
$>6.0 \mathrm{mmol} / \mathrm{L}$ on placebo $(\%)$ & $1.71(1.03-2.40)$ & $2.62(1.77-3.48)$ & $10.4(6.45-14.4)$ \\
$>6.0 \mathrm{mmol} / \mathrm{L}$ RR $(-)$ & $2.75(1.36-4.13)$ & $2.30(1.51-3.09)$ & $1.49(0.730-2.25)$
\end{tabular}

The $\leq 4.8$ limits scenario is like FIDELIO-DKD; the $\leq 5.0$ limits scenario differs by increased inclusion and uptitration limits. The subset included with $\leq 5.0$ limits but not with $\leq 4.8$ limits is shown in the final column. Data are displayed as mean (95\% prediction interval) of 30 iterations of the simulation. Hyperkalemia is calculated as the percentage of patients with one or more central laboratory observations $>5.5$ or $>6.0 \mathrm{mmol} / \mathrm{L}$ after the baseline visit

$R R$ relative risk or risk ratio of $\%$ patients with hyperkalemia with finerenone relative to placebo

benefit-risk assessment and the safe use of finerenone in patients with CKD and T2DM when guidance for dose and dose adaptions are followed.

Supplementary Information The online version contains supplementary material available at https://doi.org/10.1007/s40262-021-01083-1.

Acknowledgements The authors thank the FIDELIO-DKD committees, investigators, and patients; Sissy Stauffenberg (ClinStat), Martin Gebel, Cosima Klein, Aziz Tuermer, Jacobus Buytendach, and Patrick
Schloemer (Bayer) for derived variables, information about data, and alignment with statistical analysis; Martijn van Noort (Leiden Experts on Advanced Pharmacokinetics and Pharmacodynamics) for critical discussion of popPKPD methods and results; Udo Kuhnert and Nicholas Downie (Bayer) for supporting pharmacometric analysis dataset creation; Nurahamid Siddiki, Md. Aminul Islam, and Rumana Khatun (Shafi Consultancy) for data set quality control; Hauke Ruehs, Yang Zhang, and Alexander Solms (Bayer) for critical discussion of methods and results; Roland Heinig and Susanne Metzger for critical discussion of results; Simone Steinbach and Ui Yen Morgenthaler (Bayer) for medical writing support. 


\section{Declarations}

Funding Leiden Experts on Advanced Pharmacokinetics and Pharmacodynamics (LAP\&P) conducted the analysis as contract research for Bayer. However, LAP\&P (SCG, NS) were not paid to contribute to the manuscript preparation.

Conflicts of interest SCG and NS are employed by LAP\&P, a contract research organization working for Bayer. AS, AHB, MB, AJ, DG, JL, and TE are Bayer employees and potential shareholders of Bayer AG.

Availability of data and material Data will be made available in an electronic repository in future, at a date to be specified by Bayer.

Code availability NONMEM analysis code is provided in the ESM.

Ethics approval The study protocol was approved by international review boards, independent ethics committees, and competent authorities according to national and international regulations. FIDELIO-DKD was conducted in accordance with the ICH Harmonized Tripartite Guideline for Good Clinical Practice.

Consent to participate All study participants provided written informed consent before entering the study.

Consent for publication All study participants provided written informed consent before entering the study.

Open Access This article is licensed under a Creative Commons Attribution-NonCommercial 4.0 International License, which permits any non-commercial use, sharing, adaptation, distribution and reproduction in any medium or format, as long as you give appropriate credit to the original author(s) and the source, provide a link to the Creative Commons licence, and indicate if changes were made. The images or other third party material in this article are included in the article's Creative Commons licence, unless indicated otherwise in a credit line to the material. If material is not included in the article's Creative Commons licence and your intended use is not permitted by statutory regulation or exceeds the permitted use, you will need to obtain permission directly from the copyright holder. To view a copy of this licence, visit http://creativecommons.org/licenses/by-nc/4.0/.

\section{References}

1. Bakris GL, Agarwal R, Anker SD, Pitt B, Ruilope LM, Rossing $\mathrm{P}$, et al. Effect of finerenone on chronic kidney disease outcomes in type 2 diabetes. N Engl J Med. 2020;383(23):2219-29.

2. Bakris GL, Agarwal R, Anker SD, Pitt B, Ruilope LM, Nowack $\mathrm{C}$, et al. Design and baseline characteristics of the finerenone in reducing kidney failure and disease progression in diabetic kidney disease trial. Am J Nephrol. 2019;50(5):333-44.

3. Pitt B, Filippatos G, Agarwal R, Anker SD, Bakris GL, Rossing $\mathrm{P}$, et al. Cardiovascular events with finerenone in kidney disease and type 2 diabetes. N Engl J Med. 2021.

4. Patel V, Joharapurkar A, Jain M. Role of mineralocorticoid receptor antagonists in kidney diseases. Drug Dev Res. 2021;82(3):341-63.
5. Capelli I, Gasperoni L, Ruggeri M, Donati G, Baraldi O, Sorrenti $\mathrm{G}$, et al. New mineralocorticoid receptor antagonists: update on their use in chronic kidney disease and heart failure. J Nephrol. 2020;33(1):37-48.

6. Snelder N, Heinig R, Drenth HJ, Joseph A, Kolkhof P, Lippert J, et al. Population pharmacokinetic and exposure-response analysis of finerenone: insights based on phase IIb data and simulations to support dose selection for pivotal trials in type 2 diabetes with chronic kidney disease. Clin Pharmacokinet. 2020;59(3):359-70.

7. van den Berg P, Ruppert M, Mesic E, Snelder N, Seelmann A, Heinig R, et al. Finerenone dose-exposure-primary efficacy response in FIDELIO-DKD phase 3-population pharmacokinetic and time-to-event analysis. Clin Pharmacokinet. 2021. https://doi. org/10.1007/s40262-021-01082-2.

8. Filippatos G, Anker SD, Agarwal R, Pitt B, Ruilope LM, Rossing $\mathrm{P}$, et al. Finerenone and cardiovascular outcomes in patients with chronic kidney disease and type 2 diabetes. Circulation. 2021;143:540-52.

9. Schnider TW, Minto CF, Filipovic M. The drug titration paradox: correlation of more drug with less effect in clinical data. Clin Pharmacol Ther. 2021;110(2):401-8.

10. Mould DR, Frame B. Population pharmacokinetic-pharmacodynamic modeling of biological agents: when modeling meets reality. J Clin Pharmacol. 2010;50(9 Suppl):91S-100S.

11. Lipkovich I, Adams DH, Mallinckrodt C, Faries D, Baron D, Houston JP. Evaluating dose response from flexible dose clinical trials. BMC Psychiatry. 2008;8:3

12. Xu XS, Yuan M, Nandy P. Analysis of dose-response in flexible dose titration clinical studies. Pharm Stat. 2012;11(4):280-6.

13. Turrigiano G. Homeostatic signaling: the positive side of negative feedback. Curr Opin Neurobiol. 2007;17(3):318-24.

14. Sauro HM. Control and regulation of pathways via negative feedback. J R Soc. 2017;14(127):20160848.

15. Stelling J, Sauer U, Szallasi Z, Doyle FJ 3rd, Doyle J. Robustness of cellular functions. Cell. 2004;118(6):675-85.

16. Kovesdy CP, Matsushita K, Sang Y, Brunskill NJ, Carrero JJ, Chodick G, et al. Serum potassium and adverse outcomes across the range of kidney function: a CKD Prognosis Consortium metaanalysis. Eur Heart J. 2018;39(17):1535-42.

17. Bandak G, Sang Y, Gasparini A, Chang AR, Ballew SH, Evans $\mathrm{M}$, et al. Hyperkalemia after initiating renin-angiotensin system blockade: the Stockholm Creatinine Measurements (SCREAM) Project. J Am Heart Assoc. 2017;6(7):e005428.

18. Babazono T, Nyumura I, Toya K, Hayashi T, Ohta M, Suzuki K, et al. Higher levels of urinary albumin excretion within the normal range predict faster decline in glomerular filtration rate in diabetic patients. Diabetes Care. 2009;32(8):1518-20.

19. Savarese G, Carrero JJ, Pitt B, Anker SD, Rosano GMC, Dahlstrom U, et al. Factors associated with underuse of mineralocorticoid receptor antagonists in heart failure with reduced ejection fraction: an analysis of 11215 patients from the Swedish Heart Failure Registry. Eur J Heart Fail. 2018;20(9):1326-34.

20. Currie G, Taylor AH, Fujita T, Ohtsu H, Lindhardt M, Rossing $\mathrm{P}$, et al. Effect of mineralocorticoid receptor antagonists on proteinuria and progression of chronic kidney disease: a systematic review and meta-analysis. BMC Nephrol. 2016;17(1):127.

21. FDA. US Prescribing Information. 2021. http://www.accessdata. fda.gov/drugsatfda_docs/label/2021/215341s000lbl.pdf. Accessed 16 July 2021. 